\title{
Philosophiques
}

\section{Le modèle du vivant dans la physique cartésienne}

\section{François Duchesneau}

Volume 23, numéro 2, automne 1996

URI : https://id.erudit.org/iderudit/027405ar

DOI : https://doi.org/10.7202/027405ar

Aller au sommaire du numéro

Éditeur(s)

Société de philosophie du Québec

ISSN

0316-2923 (imprimé)

1492-1391 (numérique)

Découvrir la revue

Citer cet article

Duchesneau, F. (1996). Le modèle du vivant dans la physique cartésienne.

Philosophiques, 23(2), 389-404. https://doi.org/10.7202/027405ar d'utilisation que vous pouvez consulter en ligne.

https://apropos.erudit.org/fr/usagers/politique-dutilisation/ 


\title{
IE MODĖIE dU VIVANI DANS LA PHYSIQUE CARIÉSIENNE
}

\author{
PAR \\ François Duchesneau
}

La théorie de l'ètre vivant est sans doute l'une des parties les plus incomplètes et les plus problématiques de la philosophie de Descartes. Les grands textes cartésiens, du moins ceux que nos prédécesseurs ont jugés grands, en font presque intégralement l'économie, ou du moins ils semblent n'y consacrer que des analyses marginales. Et pourtant, comme celle de la morale, la réforme de la médecine, désormais fondėe sur des principes certains, apparaissait à Descartes l'un des fruits majeurs à attendre de la révolution théorique qu'il avait entreprise. Le monde, exposé primordial de sa physique que Descartes décide de ne pas publier en raison de la condamnation de Galilée, comportait l'explication de l'homme en tant qu'être purement corporel. Mais la théorie cartésienne de l'être vivant restera pour l'essentiel inédite du vivant de Descartes. L'homme que Descartes rédigeait vers 1632 fit lobjet d'une publication posthume (version latine en 1662, texte français en 1664). Lautre fragment majeur, La description du corps humain, comportant l'exposé de l'embryologie cartésienne et terminé en 1648, ne parut également que dans l'édition de 1664. Or, malgré ces circonstances, l'impact du modèle cartésien de l'animal-machine sera majeur. La théorie de l'ètre vivant apparaissait d'entrée de jeu comme la partie de la physique la plus irréductible à la subordination méthodologique qu'impliquait l'analyse more geometrico, celle pour laquelle les schèmes hérités de la philosophie d'Aristote et de la médecine de Galien semblaient les plus irremplaçables et les plus indestructibles. L'audace spéculative de Descartes est extrême dans cette partie, incontestablement la plus "romanesque", de sa physique. Il élabore une pluralité de modèles mécanistes afin de rendre compte des fonctions que la physiologie d'inspiration galéniste ne concevait qu'à l'aide de représentations téléologiques et rattachait à des principes d'animation et à des facultés spécifiques. Alors même que Descartes prétend produire des explications parfaitement conformes à l'intelligibilité mathématique, ces explications se trouvent déduites d'hypothèses a priori reposant sur des analogies mécaniques; s'il présume rejoindre ainsi de façon adéquate les données que l'expérience lui révèle, tant dans le cas de descriptions d'observation que dans celui d'expérimentations cruciales, la grille d'analyse des phénomènes reste le produit d'une construction fictive. Mais le paradigme d'une mathesis mechanica appliquée à la genèse et au fonctionnement de l'animal complexe jouera en fait un double róle, celui de fournir le cadre méthodologique d'explications valides pour une grande partie de la physiologie ultérieure et celui de ruiner, également pour bonne part, le recours aux propriétés et facultés relevant des âmes végétatives ou animales, principes intermédiaires entre la mens et la 
res extensa. Dans ces conditions, j'entends détailler les caractéristiques du modèle cartésien de l'animal-machine. Je l'aborderai sous deux angles: sa capacité de représenter la fonctionnalité des phénomènes vitaux; les limites de la théorisation qu'il rend possible. Puis, je présenterai le modèle mécaniste inachevé de la génération.

\section{La théorie de l'animal-machine}

La théorie cartésienne de l'animal-machine est d'abord exposée dans le traité de L'homme. Le passage suivant est significatif dans la mesure où il met en valeur le jeu multiple des modèles analogiques dans la formulation d'hypothèses explicatives :

Je suppose que le Corps n'est autre chose qu'une statue ou machine de terre, que Dieu forme tout exprès, pour la rendre la plus semblable à nous qu'il est possible : en sorte que, non seulement il lui donne en dehors la couleur et la figure de tous nos membres mais aussi qu'il met au dedans toutes les pièces qui sont requises pour faire qu'elle marche, qu'elle mange, qu'elle respire, et enfin qu'elle imite toutes celles de nos fonctions qui peuvent être imaginées procéder de la matière, et ne dépendre que de la disposition des organes. Nous voyons des horloges, des fontaines artificielles, des moulins, et autres semblables machines, qui n'étant faites que par des hommes, ne laissent pas d'avoir la force de se mouvoir d'elles-mêmes en plusieurs diverses façons; et il me semble que je ne saurais imaginer tant de sortes de mouvements en celle-ci, que je suppose être faite des mains de Dieu, ni lui atiribuer tant d'artifice, que vous n'ayez sujet de penser qu'il y en peut avoir encore davantage ${ }^{1}$.

Sans doute ce texte permet-il de comprendre le recours hypothétique aux modèles de l'être vivant suivant un double rapport au mécanisme et à la téléologie. Bien que la mème méthodologie prévale encore dans la phase ultime des réféxions cartésiennes, le recours aux analogies mécaniques, suivant l'ordre de déploiement de La description du corps humain, s'y fera plus discret, au profit d'un démarcage plus direct des modèles explicatifs sur les processus liés aux structures anatomiques. Corrélativement, les considèrations embryologiques, absentes des textes antérieurs, impliqueront alors des modèles de type génétique, à la fois originaux et extrèmement spéculatifs.

Le projet est initialement de constituer une théorie de la médecine sur les fondements de la science certaine de la Nature, de "traiter exactement de la Médecine ${ }^{2}$ ", c'est-à-dire en fonction des seules lois du mouvement appliquées à des parties de la matière, c'est-à-dire de l'étendue : celles-ci, pour ètre supposées d'une certaine figure, mème très complexe, n'en restent pas moins réductibles à une analyse faisant fond sur la seule intelligibilité géométrique. Si toute machine apparait comme configuration mobile de parties, il est indéniable que la seule mobilité dont soit susceptible la mécanique vitale, se ramène à une détermination des parties à des mouvements locaux donnés, sitôt que l'impulsion se produisant déclenche le jeu articulé des diverses pièces ou organes. La réduction des mouvements vitaux aux lois de la mécanique est bien spécifiée par Descartes, ainsi que la

I. AT XI, 120 .

2. Descartes, Lettre-préface aux Principes de la philosophie, AT IX-2, 17. 
convergence entre cette exigence d'intelligibilité géométrique de tous les phènomènes du corps vivant et la mise en évidence de la structure anatomique comme agencement de pièces permettant l'accomplissement de fonctions que l'on peut décrire comme autant de processus mécaniques ${ }^{3}$. Toute téléologie doit ètre exclue de l'étude des corps vivants, au profit de la déduction ou construction analogique des fonctions organiques à partir du seul agencement mécanique des parties, du moins toute téléologie qui impliquerait l'intervention d'ámes ou de quelques principes spirituels que ce soit, comme causes efficientes présumées du mouvement fonctionnel des parties. D'où une justification par arguments critiques du mécanisme appliqué à l'explication des fonctions vitales.

Lillustration de la nouvelle physiologie que Descartes conçoit comme l'une des parties intégrantes et l'un des aboutissements de sa physique, se prêsente excellemment dans le modèle qu'il construit pour rendre compte de la mécanique vitale sur base de circulation sanguine et dont il fournit le specimen dans la $5^{\mathrm{e}}$ Partie du Discours de la méthode $e^{4}$ à partir des analyses qu'il avait menées dans Le monde. À noter le rôle de modèle que Descartes accorde d'entrée de jeu au mouvement du cœur et des artères pour l'explication de tous les autres mouvements qui se déroulent dans l'organisme animal; c'est là " le premier et le plus général [mouvement] qu'on observe dans les animaux ${ }^{5}$ ". La base de l'analyse se fonde sur le rappel de la structure anatomique du cœur et des réseaux artériels et veineux qui s'y raccordent, avec insistance particulière sur la disposition des valvules ordonnées à un sens unique, soit centripète, soit centrifuge, de déplacement des fluides, et sur la différence de consistance des artères et des veines. À l'observation immédiate, Descartes rattache également le constat de la chaleur de l'organe cardiaque qu'il juge supérieure à celle de toutes les autres parties. La structure cardiaque et vasculaire, ainsi que la chaleur particulière de l'organe central, rend compte du mécanisme en lequel se traduisent l'altération et le mouvement du sang. Descartes suppose que deux globules de sang

3. Il n'est pas inutile de rappeler l'affirmation de Descartes dans la lettre à Buitendijck de 1643, suivant laquelle les divers genres de mouvement, impliquant ceux de la génération, de la croissance/décroissance et de l'altération qualitative se trouvent réductibles au seul mouvement local qui régit aussi bien les corps animés : cf. AT IV, 65 : "Cæterum non admitto varia motuum genera, sed solum localem, qui corporum omnium, tum animatorum, tum inanimatorum, communis est ". Il est remarquable que dans cette lettre, Descartes interprète l'âme des animaux comme étant non le mouvement en tant que tel - réalité modale - mais un composé particulier de substance corporelle, en l'occurrence le sang, en raison de ses propriétés dynamiques, analysables suivant la catégorie du mouvement : AT IV, 65 : "[...] sanguinem esse illorum animam : sanguis enim est corpus fluidum citissime motum, cuius pars subtilior dicitur spiritus, et quæ ab arteriis per cerebrum in nervos et musculos indesinenter fluens totam corporis machinam movet ".

4. Je renvoie ici volontiers à l'utile travail d'A. Bitbol-Hespériès, Le principe de vie chez Descartes, Paris, Vrin. 1990, qui éclaire sur plusieurs aspects le contexte théorique dans lequel se situe l'analyse cartésienne de l'étre vivant. Sur le mode de réception de la théorie de Harvey par Descartes et les cartêsiens, cf. R. French, William Harvey's Natural Philosophy, Cambridge, Cambridge University Press, 1994, 179-226.

5. AT VI, 46 . 
provenant de la veine cave et de la veine pulmonaire, de taille assez considérable en raison du diamètre de ces vaisseaux, s'écoulent respectivement des oreillettes droite et gauche dans les ventricules correspondants. En raison de la chaleur de l'organe et du processus de fermentation qui s'y déroule par l'effet des particules sanguines effervescentes précédemment retenues dans les fibres des parois, ces deux gouttes se raréfient et se dilatent au point d'obturer les valvules sigmoïdes et mitrales : le sang ne peut donc ètre refoulé dans les oreillettes. L'expansion du liguide sanguin entraine alors l'ouverture des valvules de l'artère pulmonaire et de l'aorte et la projection du sang dans les artères. Déplétion (systole) et expansion (diastole) entrainent les mouvements contractiles alternés des oreillettes et des ventricules et par suite la pulsation artérielle. Le modèle mécanique ainsi constitué répondrait à la "force des démonstrations mathématiques " en raison de la convergence des faits observables avec des raisons congruentes relevant des concepts géométrico-mécaniques élémentaires auxquels on peut attribuer une intelligibilité intuitive.

Au reste, afin que ceux qui ne connaissent pas la force des démonstrations mathématiques, et ne sont pas accoutumés à distinguer les vraies raisons des vraisemblables, ne se hasardent pas de nier ceci sans l'examiner, je les veux avertir que ce mouvement, que je viens d'expliquer, suit aussi nécessairement de la seule disposition des organes qu'on peut voir à l'oil dans le cœur, et de la chaleur qu'on y peut sentir avec les doigts, et de la nature du sang qu'on peut connaitre par expérience, que fait celui d'un horloge, de la force, de la situation, et de la figure de ses contrepoids et de ses roues ${ }^{6}$.

Suit alors, selon l'ordre de l'analyse, une prise en compte de la découverte de la grande circulation par Harvey : celle-ci se fonde sur l'hypothèse d'un transit du sang du réseau artériel au réseau veineux par anastomose des artérioles aux veinules. Une partie des preuves expérimentales de Harvey est reprise pour étayer le modèle mécanique de circulation perpétuelle. Mais les arguments harveyens relatifs à la contraction cardiaque et à la systole comme temps fort du processus sont omis; Descartes, nous l'avons vu, y substitue ses propres schèmes analytiques. D'ailleurs, les arguments développés par Descartes visent surtout à confirmer son hypothèse relative à la mécanique circulatoire : sans en appeler à des causes obscures du type des formes substantielles, cette mécanique se fonderait sur la chaleur cardiaque et sur l'altération des parties sanguines en structure et en mouvement ; une telle altération résulterait d'un "feu sans lumière", sorte de processus interne de fermentation, hypostasié dans la disposition organique même ${ }^{7}$. Les arguments cartésiens sont les suivants : 1) la couleur et la température

6. AT VI, 50.

7. AT VI, $46:$ "[...] et sans mettre en lui lle corps hypothétique semblable au nôtrel, au commencement, aucune âme raisonnable, ni aucune autre chose pour y servir d'âme végétante ou sensitive, sinon qu'il [Dieul excitât en son coeur un de ces feux sans lumière, que j'avais déjà expliqué [dans Le mondel, et que je ne concevais point d'autre nature que celui qui échauffe le foin, lorsqu'on l'a renfermé avant qu'il fút sec, ou qui fait bouillir les vins nouveaux, lorsqu'on les laisse cuver sur la rape *. 
différente des sangs artériels et veineux s'expliqueraient par la raréfaction et la distillation des parties sanguines dans le cœur;2) la différence de consistance des artères par rapport aux veines serait proportionnée à la qualité et à la force du sang qui s'y projette ; 3) la disparité des ventricules droit et gauche se justifierait par la plus grande subtilité et la force d'expansion supérieure du sang après une première distillation dans le ventricule droit, ainsi que par le plus court trajet de la petite circulation qui empêche une trop forte densification du sang; enfin, 4) lobservation médicale des propriétés du pouls correspondrait à l'incidence des variations de composition du sang sur le mécanisme d'altération cardiaque et ses effets moteurs ; 5) la chaleur animale pourrait être directement corrélée aux modalités de transformation du sang dans le cœur.

Une fois le modèle du mouvement cardiaque et sanguin ainsi construit, Descartes, dans le Discours de la méthode, esquisse les étapes d'une extension analogique qui permettrait d'instruire l'explication des autres fonctions de l'organisme par le déploiement de modèles similaires. C'est là le programme que L'homme tentait de réaliser et qui donnera lieu aux développements exposés dans Les passions de l'âme et La description du corps humain. Le point épistémologique le plus significatif de l'esquisse tient sans doute à l'idèe de corroborer l'hypothèse mécaniste par la convergence mème des modèles qui l'exemplifient diversement. Il s'agit là d'une forme de validation par la conjonction des faits empiriques dénombrés et l'assimilation analogique des modèles sous l'intelligibilité d'un schème unique : ce schème se fonderait sur les seules notions de propriétés géométrico-mécaniques et exclurait de l'explication toute forme ou faculté appartenant aux physiologies de provenance aristotélicienne et galénique.

Sans nul doute, si Descartes propose sa théorie mécaniste de l'ètre vivant avec le statut qu'il assigne à l'hypothèse, la comparaison avec les "automates" Iui fournit des modèles privilégiés dans le développement et la validation de cette hypothèse. De fait, les divers types d'automates constituent autant de modèles qu'on peut utiliser analogiquement pour figurer la structure et le fonctionnement de tels ou tels systèmes d'organes du corps vivant. Ce que confirme le passage de la $5^{c}$ Partie du Discours de la méthode où Descartes justifie sa théorie en alléguant :

[Elle ne semblera] nullement ètrange à ceux qui, sachant combien de divers automates, ou machines mouvantes, l'industrie des hommes peut faire, sans y employer que fort peu de pièces, à comparaison de la grande multitude des os, des muscles, des nerfs, des artères, des veines, et de toutes autres parties qui sont dans le corps de chaque animal, considéreront ce corps comme une machine qui, ayant été faite des mains de Dieu, est incomparablement mieux ordonnée et a en soi des mouvements plus admirables quaucune de celles qui peuvent ètre inventées par les hommes ${ }^{8}$.

Mais comment Descartes associe-t-il cette analogie des "automates " à la constitution de modèles propres à soutenir l'explication des fonctions vitales?

8. Descartes, Discours de la méthode, AT VI, 55-56. 
Sur ce point, il est aisé de remarquer que l'utilisation de l'analogie pour fins d'èlaboration de modèles comporte plusieurs formes. Et d'abord, Descartes compare le corps vivant dans son ensemble comme dans ses parties, à plusieurs mécanismes plus ou moins automatiques de types distincts : horloges, fontaines artificielles, moulins, orgues d'église, etc., en somme, à des systèmes qui diffèrent tant par lagencement interne des parties que par le mode de transmission des mouvements. En outre, le modèle de l'horloge sert plus précisément à représenter l'autonomie et la régularité de fonctionnement du corps vivant, indépendamment de tout principe de spontanéité inventive, indépendamment de tout "instrument universel qui [puisse] servir en toutes sortes de rencontres", telle la raison : la nature agit dans les animaux "selon la disposition de leurs organes ainsi qu'on voit qu'un horloge qui n'est composé que de roues et de ressorts, peut compter les heures, et mesurer le temps plus justement que nous avec toute notre prudence ${ }^{9}$. Les modèles hydrauliques et pneumatiques représentent les processus circulatoires du sang et des esprits animaux, qui assurent la coordination vitale. Ainsi Descartes compare-t-il le système neuro-moteur à l'orgue d'église et ses tuyaux; il le compare aussi au système hydraulique des fontaines; et les deux modèles se combinent dans le fameux passage du traité de L'homme où la circulation des esprits animaux dans le cerveau et les nerfs est interprétée suivant le mécanisme des fontaines musicales ${ }^{10}$. Des modèles plus désarticulés, ceux de simples outils mécaniques, comme la corde, le levier, le coin, la poulie, bref les machines simples dont Descartes avait fourni un petit traité à l'intention de Constantin Huygens ${ }^{11}$, semblent valoir lorsqu'il s'agit d'expliquer la transmission des sensations et la structure opératoire des muscles. Mais, lorsqu'il s'agit d'expliquer la production de force motrice, le modèle utilisé est celui d'une machine à feu, le cœur, dont le mécanisme serait déclenché par un processus chimique de fermentation, analogue à celui du foin ou du vin. Du point de vue même de Descartes, tout nous semble indiquer l'inadéquation relative des modèles à représenter le type d'agencement fonctionnel hypercomplexe en lequel un organisme animal peut consister. Par exemple, Descartes peut-il ètre dupe de

9. AT VI, 57, 59.

10. Cf. Descartes, Lhomme, AT XI, 130-131: "Or, à mesure que ces esprits entrent ainsi dans les concavités du cerveau, ils passent de là dans les pores de sa substance, et de ces pores dans les nerfs; où selon qu'ils entrent, ou mēme seulement qu'ils tendent à entrer plus ou moins dans les uns que dans les autres, ils ont la force de changer la figure des muscles en qui ces nerfs sont insérés, et par ce moyen de faire mouvoir tous les membres. Ainsi que vous pouvez avoir vu aux jardins de nos Rois, que la seule force dont l'eau se meut en sortant de sa source, est suffisante pour y mouvoir diverses machines, et mēme pour les y faire jouer de quelques instruments, ou prononcer quelques paroles, selon la diverse disposition des tuyaux qui la conduisent. Et véritablement l'on peut fort bien comparer les nerfs de la machine que je vous décris, aux tuyaux des machines de ces fontaines; ses muscles et ses tendons, aux autres divers engins et ressorts qui servent à les mouvoir ; ses esprits animaux, à l'eau qui les remue, dont le cour est la source, et les concavités du cerveau sont les regards".

11. Explication des engins par l'aide desquels on peut avec une petite force lever un fardeau fort pesant, à la suite de la lettre de Descartes à Huygens du 5 octobre 1637, AT I, 435-448. 
l'incohérence relative que représente la comparaison du feu sans flamme dans la cavité cardiaque au ressort constituant l'organe moteur de l'horloge?

À la suite de M. D. Grmek, je suggère que Descartes entrevoit un type d'automate dont aucun modèle artificiel n'existait en son temps, et pour cause, puisque, selon nos catégories contemporaines, il ne pourrait s'agir que d'un automate capable d'autorégulation, programmé pour accomplir toutes les fonctions vitales ${ }^{12}$. Toutefois, cette analogie anachronique doit être considérée avec circonspection, car, partisan de la création continuée, Descartes présuppose la constance des effets de l'acte créateur, qui se prolongent, après la création de la machine, dans les processus internes de l'automate animé : si le système est autosuffisant sur le plan de l'activité motrice différenciée, c'est que l'ingénieur transcendant continue à faire fonctionner la machine, en utilisant le mème pouvoir causal qui a produit l'agencement mème de celle-ci. Ce dètail échappe facilement aux interprètes de la théorie cartésienne de l'animal-machine, parce que la thêorie de la création continuée est elle-même ambivalente, ce qui la fait facilement tenir pour ambiguë. Le système du monde s'ordonne de lui-mème en une succession de phénomènes mécaniquement liés. Les lois de la Nature ne présupposent aucune autre téléologie que la "chiquenaude" divine; en même temps, l'enchainement des phénomènes et les lois mécaniques qui les expliquent, illustrent un acte créateur, une téléologie transcendante, qui se renouvelle à chaque instant. Ce qui vaut pour le système du monde, vaut a fortiori pour les systèmes particuliers qui en dépendent suivant l'ordre de la déduction, du simple au complexe, du principe à la conséquence, de la nature simple à la complexitè des phènomènes. En raison de l'intrication des phénomènes qu'il représente, l'organisme est un monde plus complexe que le système du ciel, mais il résulte de la mème causalité transcendante. Si l'explication de chaque fonction requiert l'intelligibilité d'un enchaînement strictement mécanique de causes et d'effets, l'explication de la structure organique mēme reste irréductible aux modèles qui incarneraient la raison mécanique de tels processus.

12. Cf. M.D. Grmek, "A survey of the mechanical interpretations of life ", in A.D. Breck \& W. Yourgrau (eds.), Biology, History, and Natural Philosophy. New York, Plenum Press, 1972, 181-195, ici 187: "If a human or animal organism is interpreted in a Cartesian way, one cannot escape the logical necessity to suppose divine intervention by the First Engineer. A complicated machine must be built by some superior Intelligence. The two possibilities can be envisaged. Let me express these in modern language : an animalmachine is either an automaton with cybernetic regulations and something like a program-tape inserted in it by the First Engineer : or it is a kind of car, or better a very complex factory, which cannot operate without permanent intelligent conduction and supervision. Descartes chose the first logical possibility, which was certainly very audacious on his part, for in his time, nothing was known of feedback circuits and program records. We can now easily understand why he was not able to express clearly all the meaning of his beast-machine analogy : he was in search for a still nonexisting mechanical model ". 


\section{Le modèle mécaniste de la génération}

Pour Descartes, le problème se révèle particulièrement crucial lorsqu'il doit foumir une explication de la génération animale suivant l'ordre des causes efficientes. Ȧ supposer que l'animal-machine soit un système de parties matérielles à fonction autorégulatrice, l'autorégulation doit intervenir à la fois sur le plan de la conservation et sur celui de la reproduction du système, car les deux plans sont liês du point de vue biologique. D'où l'importance de l'embryologie aux yeux mèmes de Descartes: il lui semblait que la solution qu'il apporterait au problème de la génération serait la pierre de touche de toute science du vivant, voire de toute physique, puisqu'il s'agissait de fournir une explication causale de la production des phénomènes les plus complexes dans leur ordre ${ }^{13}$.

Pour l'explication des corps vivants, à l'époque où il rédige Le monde, Descartes n'a pu suivre l'heureuse méthode qu'il avait utiliseee pour la physique et qui consistait à fonder l'explication des phènomènes observables sur la projection d'une genèse idéale capable d'en fournir la reconstruction théorique à partir des éléments matériels primordiaux et en soumettant ceux-ci sans ordre préalable, c'est-à-dire suivant l'hypothèse d'un chaos primitif, aux lois générales de la Nature inférables de la métaphysique. Par rapport à ce schème méthodologique, le traité de L'homme manifeste la particularité que, d'entrée de jeu, Descartes y fait abstraction de la genèse des organismes vivants: il se contente alors de postuler les structures anatomiques de l'animal à l'état achevé et de s'en donner une représentation géométrico-mécanique qui puisse s'avérer aussi congruente que possible aux organes selon leur apparence observable et leurs mouvements. Il ne s'attarde même pas à tenter de rendre compte par l'analyse des microparties organiques, dans la mesure où celles-ci sont proprement imperceptibles ${ }^{14}:$ il présume que les mouvements macroscopiques résultants manifesteront suffisamment l'ordre sous-jacent des causes pour nous permettre de construire des modèles recevables. Ainsi affirme-t-il :

[... j je ne m’arrêterai pas à vous décrire les os, les nerfs, les muscles, les veines, les artères, l'estomac, le foie, la rate, le cour, le cerveau, ni toutes les autres diverses pièces dont elle l= la machine de terre que Dieu forme tout exprès pour la rendre la plus semblable à nous qu'il est possiblel doit être composée ; car je les suppose du tout semblables aux parties de notre Corps qui ont les mèmes noms, et que vous pouvez vous faire montrer par quelque savant Anatomiste, au moins celles qui sont assez grosses pour être vues, si vous ne les connaissez déjà assez de vous-même. Et pour celles qui à cause de leur petitesse sont invisibles, je vous les pourrai plus facilement et plus clairement faire connaitre en vous parlant des mouvements qui en dépendent ${ }^{15}$.

13. Sur cette question de la théorie de la génération et de l'embryologie cartésienne, $c f$. J. Roger, Les sciences de la vie dans la pensée française du $X V M T^{e}$ siècle. La génération des animaux de Descartes à l'Encyclopédie, $2^{c}$ éd., Paris, A. Colin, 1971, 140-154 et passim

14. L'usage du microscope, faut-il le rappeler, ne se généralisera que dans la seconde moitié du XVII ${ }^{\text {, }}$ postérieurement à l'œuvre cartésienne.

15. Descartes, L'homme, AT XI, 120-121. 
Présentant les thèses du traité de L'homme, le Discours de la méthode confirmera la limitation intrinsèque de cette approche méthodologique $a$ posteriori en l'attribuant à un manque provisoire de connaissance :

De la description des corps inanimés et des plantes, je passai à celle des animaux et particulièrement à celle des hommes. Mais, pour ce que je n'en avais pas encore assez de connaissance pour en parler du mème style que du reste, c'est-à-dire en démontrant les effets par les causes, et faisant voir de quelles semences, et en quelle façon, la Nature les doit produire, je me contentai de supposer que Dieu formāt le corps d'un homme entièrement semblable à l'un des nōtres, tant en la figure exterrieure de ses membres qu'en la conformation intérieure de ses organes ${ }^{16}$.

Pour pouvoir déduire les effets suivant l'ordre, il faut que les causes aient été adéquatement conçues, soit que l'on puisse avoir directement accès aux natures correspondantes comme dans le cas de l'intuition des natures simples, soit que l'on puisse les figurer hypothétiquement mais avec des garanties suffisantes d'intelligibilité. Qu'il s'agisse de déduction pure ou d'inférences hypothéticodéductives pragmatiquement et rationnellement validées, l'engendrement des effets par les causes du simple au complexe est le seul ordre de l'investigation rationnelle qui mène à une certitude morale approchant de la certitude métaphysique. La meilleure formule de cette méthode de déduction causale nous est fournie par un texte majeur des Principes de la philosophie:

[...] nous devons juger que tout ce que [Dieu] a fait, a eu dès le commencement toute la perfection qu'il devait avoir; mais néanmoins. comme on connaîtrait beaucoup mieux quelle a èté la nature d'Adam et celle des arbres du Paradis, si on avait examiné comment les enfants se forment peu à peu au ventre des mères, et comment les plantes sortent de leurs semences, que si on avait seulement considéré quels ils ont èté quand Dieu les a créés: tout de même, nous ferons mieux entendre quelle est gènéralement la nature de toutes les choses qui sont au monde, si nous pouvons imaginer quelques principes qui soient fort intelligibles et fort simples, desquels nous fassions voir clairement que les astres et la terre, et enfin que tout le monde visible aurait pu ètre produit ainsi que de quelques semences, bien que nous sachions qu'il n'a pas étè produit en cette façon : que si nous le décrivions seulement comme il est, ou bien comme nous croyons qu'ül a été crééê.

Or, en ce qui concerne les semences des corps animés, une notion adéquate de leur structure et des modalités de leur transformation requérait plus, semble-t-il, que les connaissances dont Descartes disposait en 1637. Arguant de ce manque de connaissance qu'il estime remédiable, Descartes ramène la théorie mécanique visée de la formation au modèle de l'automate en prenant comme norme et comme base d'inférence le corps vivant tel que l'observation sensible nous le révèle à son stade de fonctionnement évolué. Or, du point de vue mēme de Descartes, ce procédé

16. Descartes, Discours de la méthode, AT VI, 45-46.

17. Descartes, Principes de la philosophie, III, § 45, AT LX-2, 124 (Principia, AT VIII- 1,100$)$. 
méthodologique suppléant une explication a posteriori à une explication a priori comporte d'évidentes limitations théoriques: 1) les inférences résultantes ne peuvent prétendre à un statut autre que purement hypothétique; elles se trouvent dépourvues des garanties rationnelles susceptibles de hausser la certitude morale au degré métaphysique; 2) inférée des phénomènes d'organisation vitale tels qu'ils se révèlent à l'observation sensible, la structure conçue du corps vivant ne peut se définir comme parfaitement détachée de toute interprétation anthropomorphique : après tout, les organes sont alors interprétés à l'aide de notions dérivant du rapport que l'homme entretient avec les instruments qu'il a créés. C'est du point de vue anthropomorphique de la construction des automates que se délimitent les organes et que se définissent leurs rapports fonctionnels; c'est aussi la mème technique analytique qu'il convient d'appliquer à la structure organique intégrée lorsqu'on tente d'expliquer son fonctionnement suivant un ensemble de déterminations motrices tant internes qu'externes.

L'exemple par excellence de cette approche méthodologique et de la théorie qu'elle permet d'édifier est foumi par la grande démonstration du traité de L'homme portant sur le mouvement cardiaque, sur la circulation sanguine et sur l'enchainement des fonctions qui en dérivent, particulièrement celles qui impliquent l'organe cérébral et les esprits animaux. C'est d'ailleurs cette mēme illustration que reproduit le Discours de la méthode et que l'on trouvera reprise sous un mode plus analytique dans La description du corps humain.

Or, dans la période postérieure à la publication du Discours de la méthode, Descartes se livre à des recherches qui auraient dū lui permettre de surmonter les déficiences méthodologiques de sa première théorie de l'être vivant en développant les considérations susceptibles de fonder une embryologie mécanique. Les Primæ cogitationes circa generationem animalium, comme d'ailleurs les Excerpta anatomica attestent d'une pluralité de tentatives - en particulier des observations sur les phases d'évolution de l'embryon de poulet après la fécondation à l'imitation des observations menées par Fabrici d'Acquapendente et publiées en $1621^{18}$. Ces tentatives aboutiront à la version provisoire de la théorie que l'on trouve dans La description du corps humain ${ }^{19}$.

Plusieurs témoignages tirés de la correspondance balisent en outre le cheminement complexe de cette recherche où Descartes a investi l'espoir d'achever adéquatement l'édifice de sa physique. Citons en particulier, au début du processus, la lettre à Mersenne du

18. Cf. Descartes, Excerpta anatomica, AT XI, 619-621; G. Fabrici d'Acquapendente, De formatione ovi et pulli tractatus accuratissimus, Patavii, Apud A. Benci, 1621 .

19. Sur le caractère provisoire des vues qui sont exposées dans La description du corps humain, $c f$. AT XI, 252-253: * Et bien que je n"aie pas voulu jusques ici entreprendre d'écrire mon sentiment touchant cette matière. à cause que je n'ai pu encore faire assez d'expériences, pour vérifier par leur moyen toutes les pensées que j'en ai eu[es] : je ne puis néanmoins refuser d'en mettre ici en passant quelque chose de ce qui est le plus général, et dont j'espère que je serai le moins en hasard ci-après de me dédire, lorsque de nouvelles expériences me donneront davantage de lumière *. 
20 février 1639 où Descartes, se fondant sur les dissections qu'il a entreprises, affirme :

[...] je n'y ai trouvé aucune chose dont je ne pense pouvoir expliquer en particulier la formation par les Causes Naturelles, tout de mème que j'ai expliqué, en mes Météores, celle d'un grain de sel, ou d'une petite étoile de neige. Et si j'étais à recommencer mon Monde, où j'ai supposé le corps d'un animal tout formé, et me suis contenté d'en montrer les fonctions, j'entreprendrais d'y mettre aussi les causes de sa formation et de sa naissance $^{20}$.

Les mêmes assertions entourent en quelque sorte les tentatives de 1648-1649 en vue de parachever l'entreprise, témoins la lettre à Élisabeth du 31 janvier $1648^{21}$ et celle, sans doute plus tardive, à un destinataire inconnu ${ }^{22}$, de mème que le texte, intermédiaire dans le temps, de l'Entretien avec Burman à la date du 16 avril $1648^{23}$.

La portée méthodologique de l'ébauche de théorie épigénétique que Descartes développe alors se trouve justement décrite par le passage relatif à l' embryologie mathématique "dans La description du corps humain :

$\mathrm{Si}$ on connaissait bien quelles sont toutes les parties de la semence de quelque espèce d'animal en particulier, par exemple de l'homme, on pourrait déduire de cela seul, par des raisons entièrement mathématiques et certaines, toute la figure et conformation de chacun de ses membres comme aussi réciproquement, en connaissant plusieurs particularités de cette conformation, on en peut déduire quelle est la semence. Mais à cause que je ne considère ici que la production de l'animal en général, et autant qu'il est besoin pour faire entendre comment toutes ses parties se forment. croissent, et se nourrissent, je continuerai seulement à expliquer la formation de ses principaux membres ${ }^{24}$.

20. Descartes, lettre à Mersenne du 20 février 1639, AT II 525.

21. Cf. Descartes, lettre à Elisabeth du 31 janvier 1648, AT V, $112:$ : [..] j'ai maintenant un autre écrit entre les mains [...] : c'est la description des fonctions de l'animal et de l'homme. Car ce que j'en avais brouillé, il y a douze ou treize ans [...] j'ai cru être obligé de le mettre plus au net, c'est-àdire de le refaire. Et mème je me suis aventuré (mais depuis huit ou dix jours seulement) d'y vouloir expliquer la façon dont se forme l'animal dès le commencement de son origine. Je dis l'animal en général : car. pour l'homme en particulier, je ne l'oserais entreprendre, faute d'avoir assez d'expérience pour cet effet ".

22. Cf. AT V, 260-261 : " Pour la description de l'animal, il y a longtemps que j'ai quitté le dessein de la mettre au net, non point par négligence, ou faute de bonne volonté, mais pour ce que j'en ai maintenant un meilleur. Je ne m'étais proposé que de mettre au net ce que je pensais connaitre de plus certain touchant les fonctions de l'animal, pour ce que j'avais presque perdu l'espérance de trouver les causes de sa formation; mais, en méditant lã-dessus, j'ai tant découvert de nouveaux pays, que je ne doute presque point que je ne puisse achever toute la Physique selon mon souhait, pourvu que j'aie du loisir et la commodité de faire quelques expériences :

23. Cf. Descartes, Entretien avec Bumnan, AT V, 170-171: "Quin etiam in ipso Animalis Tractatu, in quo hoc hieme laboravit, id animadvertit cum enim solum animalis functiones explicare vellet, vidit se id facere vix posse sine eo quod animalis conformationem ab ovo explicare necesse haberet, quam ex suis principiis, ita sequi animadvertit, ut rationes cur oculus, nasus, cerebrum. etc. essent, dare posset; et plane perspexat naturam rerum ex suis principiis ita constitutam esse, ut aliter non posset " .

24. Descartes, La description du corps humain, AT XI, 277. 
Trois points méritent ici d'être soulignès. 1) L'explication causale des organismes particuliers supposerait une connaissance intégrale des structures séminales spécifiques à ces diverses sortes d'organismes. 2) Le retour des effets déployés aux causes qui les produisent à partir des structures élémentaires est considéré possible et, semble-t-il, valide - il s'agit apparemment d'une argumentation hypothétique du type de celle qui se trouvait illustrée dans le traité de L'homme. Il est à noter cependant que les conditions de validation ne sont pas spécifiées. 3) Le dessein particulier de Descartes est de fournir non pas la théorie déductive complète établissant a priori les règles de formation des sortes particulières d'organisme, y compris les plus complexes, mais plutôt d'établir un modèle d'épigenèse mécanique de caractère général, susceptible de représenter la formation de systèmes organiques types. L'inférence que l'on serait tenté de faire consisterait à présumer que l'établissement de ce modèle fournit précisément le moyen principal pour valider la démarche a posteriori à partir des effets complexes : le modèle épigénétique général servirait de paradigme pour élaborer les modèles particuliers soustendant les hypothèses explicatives pour les divers cas d'espèce considérés. Ce que les commentateurs ont trop négligé dans ce passage, c'est la nouvelle équivalence établie entre la voie a posteriori et la voie a priori et le fait que par le biais de la recherche entreprise - et à mon avis par le biais du modèle mécanique de formation de type générique - l'inférence des raisons explicatives puisse désormais se fonder sur la connaissance de plusieurs particularités des phénomènes organiques résultant de l'épigenèse.

Evoquant, dans les Prima cogitationes, l'hypothèse de la génération spontanée, Descartes la considère possible, si l'on tient compte du rôle de la chaleur fermentative agissant sur des particules de disposition diverse au sein de corps organiques en putréfaction, certaines particules étant plus subtiles à l'instar de celles qui composent les esprits animaux, les autres étant plus grossières comme celles qui composent le sang ${ }^{25}$.

Mais l'essentiel de l'attention est accordé à la génération requérant le mélange des semences en provenance des deux sexes. Reprenant la vieille thèse hippocratique du defluxus, Descartes suppose que ce mélange informe comporte des particules émanant des divers organes des géniteurs et issues des fluides sanguins. Sous l'effet de la chaleur dans la matrice, se produisent des rassemblements et des structurations particulières de particules affines, les plus subtiles créant des foyers de mouvement analogues, peut-on supposer, aux tourbillons du second élément sous l'effet des déterminations motrices affectant les particules d'éther - dans les Primæe cogitationes, il est fait allusion à des tourbillons qui seraient circulaires dans le cas de la génération des plantes, et sphériques dans celui de semences animales ${ }^{26}$. Par pression, les particules

25. Descartes, Primæe cogitationes circa generationem animalium, AT XI, 505-506.

26. AT XI, 534: "In eo convenit formatio plantarum et animalium, quod fiant a partibus materiæ vi caloris in orbem convolutæ sed in hoc discrepant, quod partes materiæ, ex quibus plantæ generantur, volvantur tantum in orbem circulariter, eæ vero, ex quibus animalia, volvantur spherice et in omnes partes . 
moins subtiles en viennent alors à composer diverses structures sous l'effet de la fermentation vitale. Ainsi, suivant la version ultimement retenue, se forment progressivement à partir du cœur assumant la fonction de primum movens, les diverses structures organiques, cerveau, poumon, foie, etc., ainsi que les vaisseaux en réseaux circulaires qui les relient de façon à former des systèmes de plus en plus intégrés. Ce qui caractérise les séquences de processus dans l'analyse qu'en donne Descartes ${ }^{27}$, c'est le fait que la description morphogénétique se trouve constamment sous-tendue par des modèles mécaniques où interviennent la figuration géométrique des dispositifs organiques et l'efficience causale du mouvement agissant sur une gamme très diversifiée de particules : la typologie ouverte de ces élements corpusculaires permet de concevoir autant de dispositifs structuro-fonctionnels - selon les besoins de l'explication, pourrait-on dire, et par delà toute complexification structurale conçue more geometrico ${ }^{28}$. Jacques Roger a fait remarquablement ressortir la polyvalence de telles constructions en soulignant à quel point elles se révèlent ad hoc dans le contexte d'une recherche d'explication touchant les phénomènes hypercomplexes de l'embryogenèse ${ }^{29}$. Par audace spéculative, La description du corps humain et autres ébauches relatives à la théorie de la génération tendent en fait à rétablir par le biais de multiples dispositifs corpusculaires fonctionnels une théorisation axée sur les processus finalisés accomplis par les diverses structures et que la physiologie antérieure rattachait volontiers à des propriétés ou facultés des âmes végétative et sensitive.

La teneur de l'embryologie cartésienne est, pourrait-on dire, essentiellement programmatique et ne saurait à elle seule constituer la réforme radicale de la théorie de l'être vivant que réclameraient les normes mëthodologiques d'une véritable mathesis appliquée à l'ordre complexe des phénomènes vitaux. C'est pourquoi ultimement Descartes ne parvient pas à se distancer de la formule hypothéticodéductive imparfaite impliquant une pluralité de figurations analogiques qu'il avait d'abord mise en œuvre dans le traité de L'homme comme partie sui generis de sa physique. Il ne réussit pas à surmonter ce paradoxe consistant à soumettre la théorie physiologique à la rigueur de l'explication mécaniste qui doit prévaloir sur le territoire entier des phénomènes physiques, tout en décrivant les réalités biologiques comme des réalités de type spécial, comparables aux automates de production humaine, bien qu'à un degré de complexité et d’artifice considérablement supérieur. La principale

27. Cf. Descartes, La description du corps humain, AT XI, 253-286.

28. $C f$. le jugement très négatif posé par Emily Grosholz sur la modélisation mathématique à laquelle Descartes prétend recourir dans sa physiologie. cf. E. Grozholz, Cartesian Method and the Problem of Reduction, Oxford, Clarendon Press, 1991, 119: " I... Descartes bullds up a highly enriched version of material particles which far surpasses the earlier model of unity in virtue of geometrical shape or common motion: and he substitutes for the mechanism of impact a series of increasingly sophisticated relational structures which share the concept of machine only by the most elastic of analogies *.

29. Cf. J. Roger, Les sciences de la vie dans la pensée française du XVII siècle, 148-149. 
déficience de la théorie cartésienne résulte d'une dépendance trop étroite par rapport au modèle des automates, alors que ceux-ci sont à coup sūr déficients en ce qui a trait au pouvoir d'autorégulation, aux processus de croissance, de décroissance, de conservation et de reproduction, et qu'ils sont incapables de représenter adéquatement la vie propre des parties. Il faudra en particulier une subversion du modèle cartésien construit suivant le type d'une source centrale de force motrice, pour que puisse se développer une véritable théorie de la fibre vivante, embryon d'une conception parcellarisée de la vie organique, qui prendra son essor au début du XVIII ${ }^{e}$ siècle $^{30}$. Surtout, à considérer la physiologie cartésienne en son état méthodologiquement inachevé, sans épigenèse mécanique validée, l'hypothèse de l'animal-machine ne semble pouvoir se soutenir et satisfaire à l'explication des phénomènes que par recours à une forme de téléologie.

Je reviendrai pour conclure à la caractérisation des hypothèses dans les Principia philosophize. Nous pensons avoir trouvé le vrai chiffre d'un cryptogramme lorsque nous en voyons la signification suivre de la lecture à l'aide de la grille que nous avons forgée pour les besoins de la cause. De même, l'hypothèse d'automates formès à l'imitation des corps vivants et impliquant un artifice divin, vaut comme substitut d'une déduction en règle pour autant qu'elle permet d'édifier une théorie cohérente représentant de façon unifiée les fonctions vitales dans ce qu'elles ont de spécifique par rapport aux autres phénomènes physiques. Mais si le déchiffrement d'un message codé suppose la possession d'une grille de lecture, cette clé d'interprêtation doit elle-même répondre à des règles de formation, à des principes d'ordre susceptibles d'en exprimer la genèse rationnelle. Chez Descartes, les modèles de cette genèse rationnelle à l'arrière-plan de l'hypothèse physiologique restent à l'état d'ébauches et de constructions schématiques. Dans cet état de carence, l'hypothèse de l'animal-machine intègre sur le mode provisoire des modèles d'automatismes renvoyant à un certain état des connaissances. La norme d'intelligibilité qui intervient dans le façonnement de l'hypothèse est, dans ce cas, celle d'une sorte d'" anthropomorphisme technologique "qui prend appui sur une tẻléologie instrumentale. La tentative était brillante, mais épistémologiquement fragile, et elle ne laissait pas de favoriser de notables distorsions dans la prise en compte des données d'expérience saisies à travers un tel prisme. Les analogies disponibles à partir desquelles Descartes construit les modèles mécaniques servant à incarner la théorie des fonctions vitales, tendent à ne représenter qu'un équivalent instrumental pauvre de telles fonctions. Ces contingences ne doivent pas faire oublier toutefois l'impact indéniable des schèmes analytiques dont Descartes se fait le protagoniste en développant son modèle mécaniste de l'être vivant. Peu après la publication posthume du traité de L'homme, Niels Stensen en témoigne dans le Discours

30. Cf. M. D. Grmek, "La notion de fibre vivante chez les médecins de l'école iatrophysique ". Clio Medica, 5 (1970), 297-318. Ce thème est longuement analysé dans $F$. Duchesneau, La physiologie des Lumières, La Haye, M. Nijhoff, 1982. 
sur l'anatomie du cerveau qu'il prononce en 1665 et publie quelques années plus tard:

Pour ce qui est de Monsieur Descartes, il connaissait trop bien les défauts de l'histoire que nous avons de l'homme, pour entreprendre d'en expliquer la véritable composition. Aussi n'entreprend-il pas de la faire dans son Traité de l'homme, mais de nous expliquer une machine qui fasse toutes les actions dont les hommes sont capables. Quelques-uns de ses amis s'expliquent ici un peu autrement que lui ; on voit pourtant au commencement de son ouvrage qu'il l'entendait de la sorte ; et dans ce sens on peut dire avec raison, que Monsieur Descartes a surpassé les autres Philosophes dans ce Traité dont je viens de parler. Personne d'autre que lui n'a expliqué mécaniquement toutes les actions de l'homme, et principalement celles du cerveau ; les autres nous décrivrent l'homme même : Monsieur Descartes ne nous parle que d'une machine qui pourtant nous fait voir l'insuffisance de ce que les autres nous enseignent, et nous apprend une méthode de chercher les usages des autres parties du corps humain, avec la mème évidence qu'il nous démontre les parties de la machine de son homme, ce que personne n'a fait avant lui $[\ldots]^{3 !}$

Le rôle paradigmatique de l'animal-machine tient à la figuration mécaniste des processus fonctionnels selon un système intégré de mouvements vitaux. Au centre du modèle, se trouvent la circulation sanguine et celle des esprits animaux qui en dérive ; l'explication causale tient à l'idée d'un principe de mouvement exerçant son action par des processus chimiques et zymotiques qui se déroulent dans la texture fibrillaire du cœur. Aux arguments harveyens relatifs à la contraction cardiaque et à la systole comme temps fort du processus, Descartes oppose une mécanique circulatoire qui se fonde sur la chaleur cardiaque et sur l'altération des parties sanguines en composition et en disposition motrice. Sur cette base et par extension analogique, Descartes conçoit l'explication des autres fonctions vitales et animales d'après des modèles mécaniques diversifiès. La validité de l'explication dépendrait de la convergence des modèles et de leur capacité de transposer les données biologiques more geometrico, court-circuitant ainsi tout recours aux formes et facultés des théories physiologiques selon la tradition aristotélico-galéniste. Mème lorsqu'il s'inspire des analyses traditionnelles, Descartes évacue de la représentation théorique toute causalité de type psychomorphique - d'où la confrontation significative que traduit entre autres la correspondance avec Plempius. La théorie de l'animalmachine se structure selon le principe d'une autonomie mécanique des systèmes intégrés et centralement contrôlés : ceux-ci accompliraient les processus fonctionnels que l'on attribue à tort à la sensibilité et à la volition d'une âme animale. Si cette théorie cartésienne ne consistait qu'en simples représentations hypothétiques sur base de projections analogiques, la valeur méthodologique de tels modèles résiderait dans la capacité qu'ils confèrent de déchiffrer more geometrico l'ordre des phénomènes organiques.

31. N. Sténon, Discours sur l'anatomie du cerveau, Paris, Chez Robert de Ninville, 1669, 12-13, fac-simile in N. Steno, A Dissertation on the Anatomy of the Brain, Copenhagen, Nyt Nordisk Forlag (Arnold Busck), 1950. 
Reste alors la difficulté principielle de déterminer une modélisation mécaniste qui rende adéquatement compte de la formation même des organismes. Descartes n'avait pu surmonter cette difficultè à l'époque où il rédigeait L'homme et le Discours de la méthode. Il s'était contenté de présumer d'une èpigenèse suffisante à produire le vivant intégré et complexe que l'observation révèle au terme du processus morphogénétique. Par la suite, à la dernière époque de sa carrière, la quête d'une explication causale conforme aux normes de l'analyse suscitera des constructions hypothétiques diverses où domine le schème d'un mélange des semences animales avec composition consécutive de structures organiques sous l'effet de la fermentation vitale. L'explication alors envisagée aurait permis de produire des modèles mécanistes correspondant aux phases de la morphogenèse : ces modèles auraient intégré la représentation de dispositifs corpusculaires fonctionnels, aptes à engendrer les structures de niveau supérieur par le simple jeu des agencements organiques primordiaux et des mouvements que des facteurs externes y détermineraient. Tel est le profil épistémologique des fictions explicatives auxquelles Descartes souhaitait soumettre la recherche embryologique et sur lesquelles devait causalement reposer son modèle hypothétique de la mécanique vitale.

Département de philosophie Université de Montréal 